\title{
Optimal Power Control Technique for a Wireless Sensor Node: A New Approach
}

\author{
R. Maheswar, and R. Jayaparvathy
}

\begin{abstract}
A critical issue in wireless sensor networks is the limited availability of power and hence optimizing power is very important. In any sensor node, most of the power is consumed during packet transmission. We propose a new scheme to reduce the power consumption of nodes in a sensor network during packet transmission based on queue threshold. We develop an analytical model of a sensor node and analyze the performance of the proposed scheme in terms of performance parameters such as average power consumption and mean delay. We also derive the expression for the optimal value of threshold. Results show that the average power consumption of a node reduces by $68 \%$ for the optimal threshold value when compared to no threshold condition by considering mean arrival rate per node of 2 and there exists trade-off between the average power consumption and mean delay. We perform simulations and the results obtained show that the analytical results match with the simulation results thus validating the analytical model.
\end{abstract}

Index Terms-Average power consumption, mean delay, queue threshold, wireless sensor network.

\section{INTRODUCTION}

Wireless Sensor Networks (WSNs) have broad applications like environment monitoring, target tracking and surveillance. The unique characteristics of WSNs such as limited bandwidth, computing capacity, data delivery delay and severe energy constraints make their design more challenging. A critical issue in wireless sensor networks is the limited availability of power within the net work and hence optimizing power is very important. There are two major techniques for maximizing the sensor network lifetime: the use of energy efficient routing and the introduction of sleep/active modes for sensors \[1]. J. Carle et al. presented a good survey in $\backslash[2]$ on energy efficient area monitoring for sensor networks. The authors have observed that the best method for conserving energy is to turn off as many sensors as possible, while still keeping the system functioning.

An analytical model was presented in [3] to analyze the system performance in terms of network capacity, power consumption and data delivery delay, against the sensor dynamics in on/off modes. Most existing work on sensor networks consider homogeneous sensor networks where all

R. Maheswar is with VLB Janakiammal College of Engineering and Technology, Coimbatore, India (phone: 91-422-2604567; fax: 91-4222607152; e-mail: maheshh3@ rediffmail.com).

R. Jayaparvathy is with Coimbatore Institute of Technology, Coimbatore, India (e-mail: jaya_parvathy@yahoo.com). sensor nodes are assumed to have the same capabilities. However, a homogeneous ad-hoc network has poor fundamental limits and performance. P. Gupta and P. R. Kumar have demonstrated the performance bottleneck of homogeneous ad-hoc network via theoretical analysis in [4], simulation experiments and test bed measurements [5]. Several recent papers have studied Heterogeneous Sensor Networks (HSNs) and these literatures showed that HSNs can significantly improve sensor network performance [6]. Since the power consumed by the individual nodes in the sensor network that affects the network lifetime is very crucial in all WSN applications, different algorithms for minimizing the power consumption show that there exists trade-offs between the energy consumption and the data delivery delay [7].

In this paper, we propose a new power minimization scheme by which the average power consumption of individual nodes in the sensor network is reduced during packet transmission based on queue threshold. We develop an analytical model for analyzing the system performance in terms of average power consumption and mean delay. To the best of our knowledge, this is the first analytical model that specifically represents the sensor node's behaviour in IDLE state and BUSY state during its period of active time based on queue threshold to investigate the network performance in terms of mean delay and average power consumption.

The rest of this paper is organized as follows. In section II, we present the system model. In section III, we present the performance analysis and provide numerical solutions for determining the mean delay, average power consumption of a sensor node and the optimum threshold value of $N$. Section IV describe the simulation model and the results and discussion are presented in section V. In section VI, we provide the conclusions.

\section{SYSTEM MODEL}

We consider a sensor network composed of stationary and identical nodes which are uniformly distributed and the sink node collecting all information gathered by the sensors is located at the centre in the field. The two major operational states of a sensor node are sleep state and active state. The sleep state corresponds to the lowest value of the node power consumption; while being asleep, a node cannot interact with the external world. During active state, a node may be in idle mode or it may generate data or it may transmit and/or receive data packets. Apart from the above said operational states, we define two sub-operational states during active state namely, IDLE state and BUSY state as shown in Fig. 1. During IDLE 
state, the node will be in idle mode or receive mode or the node may generate data but the node will not be in transmit mode. During BUSY state, the node will be in transmit mode but the node will not in idle mode. In BUSY state, it is assumed that a node can transmit, receive and generates data simultaneously [3].

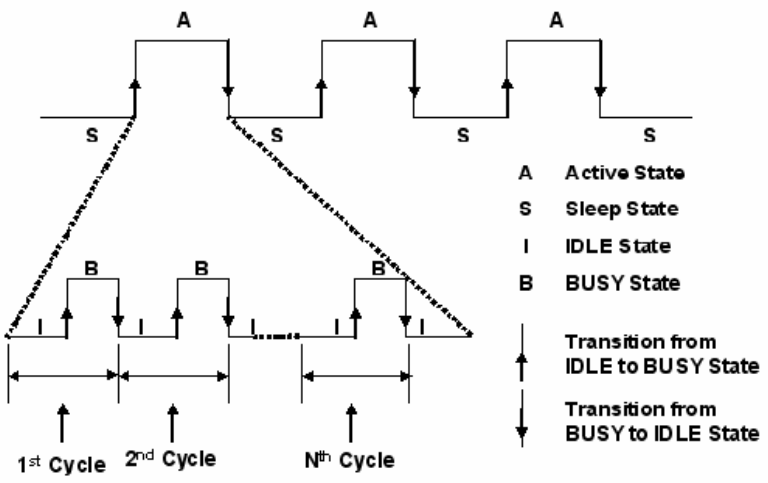

Fig. 1. Sub-operational states of a sensor node during active state.

The following assumptions are made for analysis:

- All sensor nodes in the network are identical

- Arrival rate corresponds to the data generated at the sensor nodes

- The arrival of data packets to sensor nodes is assumed to follow a Poisson process with mean arrival rate per node $(\lambda)$

- Packets are delivered from sensor node with mean service time $(1 / \mu)$

- There is no channel contention

- Buffer is assumed to be empty whenever the node switches from sleep to active state

All the sensor nodes during its period of active time will be in IDLE state or BUSY state. Here, a sensor node, during its period of active time, remains in IDLE state and switches to BUSY state when the node's buffer is filled at least with threshold number of packets $(N)$ i.e., queue threshold and the node switches back from BUSY state to IDLE state when there are no packets in the buffer. During BUSY state, the nodes deliver the packets to its neighbourhood node or to the sink node. Such switching actions between IDLE state to BUSY state and BUSY state to IDLE state are referred to as transitions.

The average power consumption of a node depends on the queue threshold since most of the energy is consumed during transmission i.e., during BUSY state. We also determine optimal threshold value $\left(N^{*}\right)$ of $N$ for which the sensor nodes consume very less power. Since the focus of this work is to minimize the power consumption of individual sensor nodes in the network during its period of active time based on queue threshold, we analyse the behaviour of a single sensor node.

\section{PERFORMANCE ANALYSIS}

In this section, we analyse the behaviour of a single sensor node. As mentioned in section II, the arrival of data packets to sensors follows a Poisson process with mean arrival rate per node $(\lambda)$ and a sensor during its period of active time, remains in IDLE state and switches to BUSY state when the sensor node's buffer is filled at least with threshold number of packets $(N)$ and switches back from BUSY state to IDLE state when there are no packets in the node's buffer. We analyze the performance of the system in terms of the following parameters.

\section{A. Mean Delay}

Mean delay experienced by the packets in a sensor node is defined as the average waiting time of the packets in the queue. To determine the mean delay experienced by the packets in a node, the two-state transition diagram of a sensor node that switches from IDLE state to BUSY state and viceversa as shown in Fig. 2 is considered.

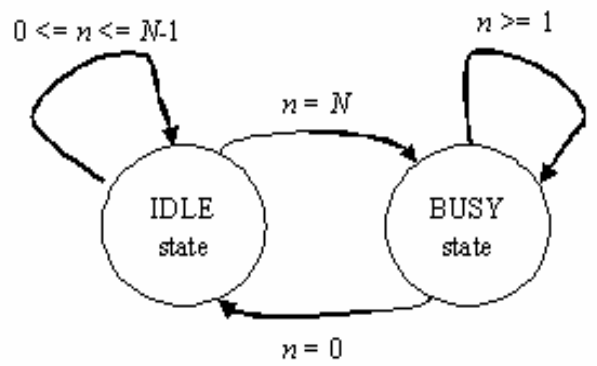

Fig. 2. Two-state transition diagram of a sensor node in IDLE state and BUSY state

Let,

$n \quad$ Number of packets in the sensor node's buffer

$N \quad$ Threshold number of packets or queue threshold

$M \quad$ Number of sensor nodes in a network

$\lambda \quad$ Mean arrival rate per node (packets/unit time)

$1 / \mu \quad$ Mean service time (unit time)

$L \quad$ Mean number of packets in the sensor node's buffer

$W_{q} \quad$ Mean waiting time of the packets in the queue (unit time)

In a network comprising of ' $M$ ' identical sensors, let the mean arrival rate per node is given by $\lambda$. The steady state balance equations obtained for the analytical model as shown in Fig. 2 are given by equations from (1) to (6).

$$
\begin{aligned}
& \lambda P_{I}(0)=\mu P_{B}(1) \\
& \lambda P_{I}(n)=\lambda P_{I}(n-1) ; 1 \leq n \leq N-1 \\
& (\lambda+\mu) P_{B}(1)=\mu P_{B}(2) \\
& (\lambda+\mu) P_{B}(n)=\mu P_{B}(n+1)+ \\
& \lambda P_{B}(n-1) ; n \geq 2 \& n \neq N \\
& (\lambda+\mu) P_{B}(N)=\mu P_{B}(N+1)+ \\
& \lambda P_{B}(N-1)+\lambda P_{I}(N-1)
\end{aligned}
$$

From the above equations, the steady state probability that the sensor is in IDLE state $P_{I}$ is determined as,

$P_{I}=1-\rho$

where,

$\rho=\frac{\lambda}{\mu}$ 
The steady state probability that the sensor is in BUSY state $P_{B}$ is determined as,

$$
P_{B}=\rho
$$

The mean number of packets in the sensor node's buffer $(L)$ is given by

$$
L=\frac{N-1}{2}+\frac{\rho}{1-\rho}
$$

The mean waiting time of the packets in the queue $\left(W_{q}\right)$ is given by,

$$
W_{q}=\frac{N-1}{2 \lambda}+\frac{\rho}{\mu-\lambda}
$$

\section{B. Average Power Consumption of a Sensor Node}

During active time, the sensor node remains in IDLE state when the number of packets is less than the queue threshold. When the threshold value is reached due to the arrival of packets, the sensor node switches from IDLE state to BUSY state and transmits a preamble packet. Preamble packet is used for synchronization of a sensor node with the nearby node or sink node for the packet transmission [8]. After synchronization, the sensor node transmits all packets to nearby node or sink node from its buffer and switches back from BUSY state to IDLE state when the buffer gets empty. Hence a cycle during the active time of a sensor node constitutes,

- Arrival of data packets

- Transition from IDLE state to BUSY state

- Synchronization

- Transmission of packets

- Transition from BUSY state to IDLE state

The sensor node, during its period of active time undergoes many such cycles depending upon the value of $N$. To determine the average power consumption of a sensor node during its period of active time, we consider the following parameters that are associated with the power consumption of a sensor node during transmission:

$C_{H} \quad$ Power consumption due to transmission of a packet per cycle in watts-sec

$C_{T} \quad$ Power consumption due to transitions and synchronization per cycle in watts-sec

$P(N) \quad$ Average power consumption of a node per unit time as a function of $N$ in watts-sec

$E[I] \quad$ Average duration of time the sensor node is in IDLE state

$E[C] \quad$ Average duration of cycle

$N_{c y} \quad$ Number of cycles per unit time

The value of queue threshold has significant effect on the number of cycles per unit time $\left(N_{c y}\right)$. The $N_{c y}$ includes arrival of data packets, transitions, synchronization and transmission of packets. Since the focus of this paper is to reduce the average power consumption based on queue threshold, the parameter that consumes power during transitions, synchronization and transmission are alone considered i.e., $C_{T}$ power consumed due to transitions and synchronization and $C_{H}$ power consumed during transmission. The power consumed during sleep state, the power consumed during idle mode and receive mode and the power consumed during data generation in active state are not considered because the power consumed due to the above said operations do not result in any power saving when no threshold condition $(N=1)$ is considered.

Probability that the sensor node in IDLE state $\left(P_{I}\right)$ can be defined as the ratio of the average duration of time the sensor node in IDLE state to the average duration of cycle. Therefore, $P_{I}$ can be written as,

$P_{I}=\frac{E[I]}{E[C]}$

where $E[I]$ can be expressed as,

$E[I]=\frac{N}{\lambda}$

Using (6) and (12) in (11), we get

$E[C]=\frac{N(1-\rho)+\rho^{k+2}\left(1-\rho^{-N}\right)}{\lambda(1-\rho)^{2}}$

The number of cycles per unit time $\left(N_{c y}\right)$ is given by

$N_{c y}=\frac{1}{E[C]}$

Using (13) in (14), we get

$N_{c y}=\frac{\lambda(1-\rho)^{2}}{N(1-\rho)+\rho^{k+2}\left(1-\rho^{-N}\right)}$

Now, the average power consumption per node $P(N)$ can be expressed as,

$P(N)=C_{H} L+C_{T} N_{c y}$

By substituting (9) and (15) in (16), the average power consumption of a sensor node per unit time $P(N)$ is obtained and it is given by

$P(N)=C_{H}\left(\frac{N-1}{2}+\frac{\rho}{1-\rho}\right)+C_{T}\left(\frac{\lambda(1-\rho)}{N}\right)$

\section{Optimal Threshold value ( $\left.N^{*}\right)$ of $N$}

The optimal threshold value $\left(N^{*}\right)$ of $N$ based on equation (17) is determined to find the value of $N$ for which the sensor node consumes minimum power and it is given by

$N^{*}=\sqrt{\frac{2 C_{T} \lambda(1-\rho)}{C_{H}}}$

\section{SIMULATION MODEL}

In this section, we present the simulation model. We consider Mica2 motes forming a sensor network. We perform the simulation for a sensor network using the various parameters given in [8]. The various network parameters and the power consumption parameters of Mica2 mote sensors used for the simulation model are given in Tables I, II and III. The values of $C_{T}$ and $C_{H}$ from Tables I, II and III are determined as $C_{T}=6.9$ mwatts-sec and $C_{H}=0.8$ mwatts-sec. Simulations results are obtained for various scenarios by varying the mean arrival rate per node and threshold number of packets to determine the number of cycles per second, 
average power consumption of an node per unit time and the mean delay experienced by the packets per node in a network.

TABLE I

NETWORK SPECIFICATIONS

\begin{tabular}{|l|l|}
\hline Mean arrival rate per node (packets/sec) & 2 to 20 \\
\hline Mean service time per packet (msec) & 15 \\
\hline Number of sensor nodes in a network & 50 \\
\hline Threshold number of packets & 1 to 20 \\
\hline
\end{tabular}

TABLE II

TIME AND CURRENT SPECIFICATIONS OF MICA2 MOTE

\begin{tabular}{|l|l|c|}
\hline Operation & Time $(\mathrm{sec})$ & $\mathrm{I}(\mathrm{mA})$ \\
\hline Initialize radio & $350 \mathrm{E}-6$ & 6 \\
\hline Turn on radio & $1.5 \mathrm{E}-3$ & 1 \\
\hline Switch to Transmit mode & $250 \mathrm{E}-6$ & 15 \\
\hline Transmit 1 byte & $416 \mathrm{E}-6$ & 20 \\
\hline
\end{tabular}

TABLE III

POWER AND OTHER SPECIFICATIONS

\begin{tabular}{|l|c|}
\hline Battery (V) & 3 \\
\hline Data rate (Kbaud) & 38.4 \\
\hline Preamble length (bytes) & 271 \\
\hline Packet length (bytes) & 36 \\
\hline Radio off $(\mu \mathrm{A})$ & 20 \\
\hline
\end{tabular}

The simulations were performed for 100 runs and a confidence interval of $99.11 \%$ was obtained. Simulation results show that the average power consumption is reduced by increasing the threshold value $N$ and the minimum power is consumed for optimal threshold value $N^{*}$ and also the results clearly show that there exists trade-offs between the energy consumption and the mean delay.

\section{RESULTS \& DISCUSSION}

In this section, we present the simulation and analytical results. By assuming the network and power consumption parameters as mentioned in Tables I, II and III, simulation and analytical results are taken to find the mean delay and the average power consumption of a node per unit time by varying the queue threshold $(N)$ and it is shown in Fig. 3 and Fig. 4. From Fig. 3, it is inferred that the mean delay increases linearly as queue threshold $(N)$ increases.

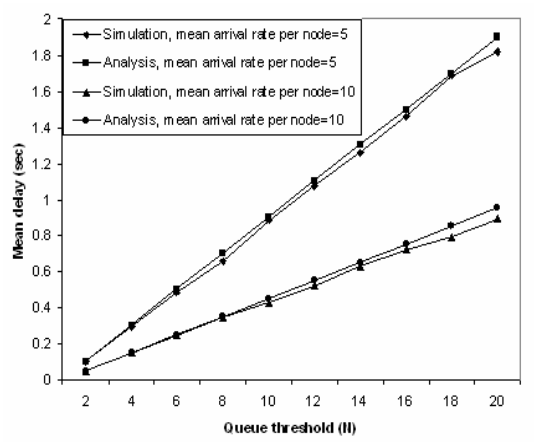

Fig. 3. Queue threshold $(N)$ vs mean delay (sec).
Fig. 3 shows the mean delay for various $N$ assuming mean arrival rate per node as 5 and 10 . The average power consumption per node is determined for $N=1, N=5$ and $N=$ 10 by assuming the mean arrival rate per node as 5 and 10 and it is shown in Fig. 4.

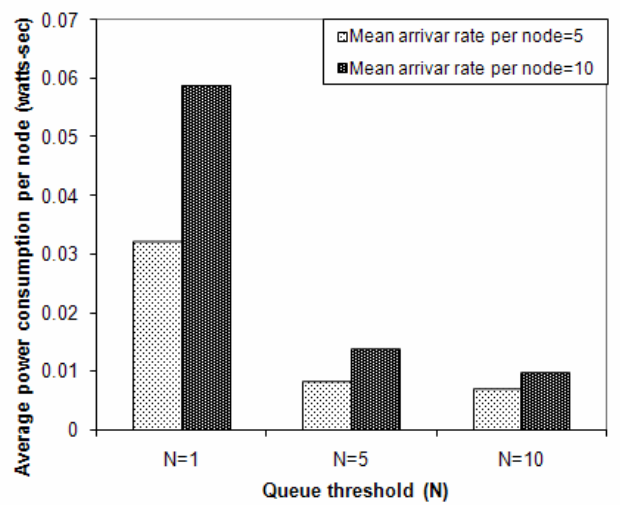

Fig. 4. Queue threshold $(N)$ vs average power consumption per node (wattssec).

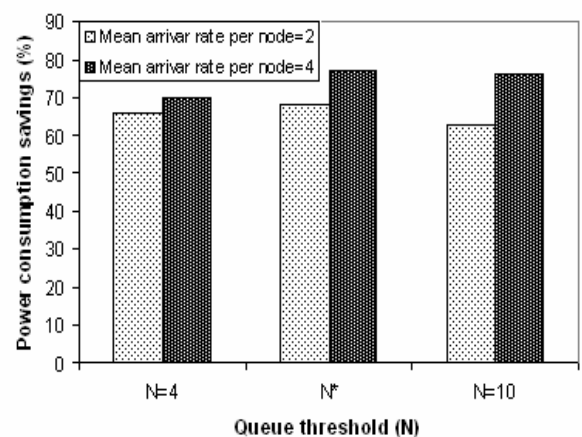

Fig. 5. Mean arrival rate/ node vs power consumption savings (\%).

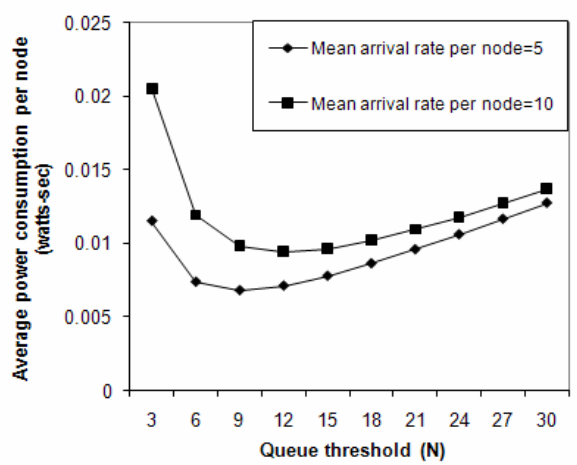

Fig. 6. Queue threshold $(N)$ vs average power consumption / node (watts$\mathrm{sec}$ ) showing minimum power consumption at optimal threshold.

From Fig. 4, it is inferred that the average power consumption per node decreases as $N$ increases. The average power consumption per node decreases as $N$ increases because, as $N$ increases, the number of cycles per second decreases resulting in less power consumption. From Fig. 3 and Fig. 4, it is also observed that the introduction of queue threshold reduces the average power consumption when compared to no threshold condition but increases the mean delay and hence there exist trade-off between the mean delay 
and the average power consumption per node with respect to the value of $N$. Fig. 5 shows the power consumption savings (\%) for different values of $N$. By assuming $N=4, N=6$ and $N$ $=10$ and mean arrival rate per node $=2$, the power consumption savings $(\%)$ is determined and it is found to be $66 \%, 68 \%$ and $63 \%$ respectively when compared to no threshold condition (i.e., $N=1$ ). The optimal threshold value $\left(N^{*}\right)$ using equation (18) for mean arrival rate per node of 2 is 6. From Fig. 5, it is also clear that the maximum power savings of $68 \%$ is achieved for the optimal threshold value $\left(N^{*}\right)=6$. Fig. 5 also shows that, when the mean arrival rate per node increases, the power consumption saving is increased and this is due to the reason that, as the arrival rate increases, the number of transitions are very high under no threshold condition when compared to introduction of queue threshold.

Considering $\lambda=5$ and $10, C_{T}$ and $C_{H}$ values as mentioned in section IV, the optimal threshold value $\left(N^{*}\right)$ using equation (18) is determined as $N^{*}=9$ and 12 respectively. The average power consumption of a sensor node is determined for various values of $N$ and it is found that the minimum power is consumed for optimal threshold value $N^{*}=9$ and 12 and it is shown in Fig. 6.

Thus from the various results obtained, it is concluded that the introduction of queue threshold $(N)$ to make the sensor node to switch from IDLE state to BUSY state has significant effect with respect to the mean delay and the average power consumption of the sensor nodes. The results clearly indicates that the average power consumption per node can be reduced to a large extent by selecting an optimal threshold value $\left(N^{*}\right)$. Clearly, the trade-offs that exists between the energy consumption and mean delay is explored through the obtained results. The simulations were performed for 100 runs and a confidence interval of $99.11 \%$ was obtained and hence the results show that our analytical results present an excellent matching with simulation results under various scenarios validating the accuracy of our approach.

\section{CONCLUSION AND FUTURE WORK}

In this work, we have proposed a new power minimization scheme by which the average power consumption of individual nodes in the sensor network is reduced based on queue threshold during its period of active time. We have developed an analytical model of a sensor node and the system performance in terms of average power consumption and mean delay have been determined. The results show that the average power consumption reduces by $68 \%$ for the optimal threshold value when compared to no threshold condition for mean arrival rate per node of 2 and there exists trade-off between the average power consumption and mean delay. The results also show that our analytical results present an excellent matching with simulation results under various scenarios showing the accuracy of our approach.

Although our work mainly focuses on average power consumption and mean delay, the level of abstraction of the proposed model is such that it can be applied to investigate various aspects in the design of sensor networks. To the best of our knowledge, this is the first analytical model that specifically represents the sensor node's behavior in IDLE state and BUSY state during its period of active time based on queue threshold to investigate the network performance in terms of mean delay and average power consumption. The model can be extended by taking channel contention into account and also the design of energy efficient routing algorithm will be another interesting area of future research.

\section{REFERENCES}

[1] Zhi Quan, Ananth Subramanian and Ali H. Sayed, "REACA: An Efficient Protocol architecture for Large Scale Sensor Networks," IEEE Transactions on Wireless Communications, vol. 6, no. 10, October 2007, pp. 3846-3855.

[2] J. Carle and D. Simplot-Ryl, "Energy-efficient area monitoring for sensor networks," IEEE Computer, vol. 37, no. 2, Feb. 2004, pp. 40-46.

[3] C. F. Chiasserini and M. Garetto, "An analytical model for wireless sensor networks with sleeping nodes," IEEE Trans. Mobile Computing, vol. 5 , no. 12 , Dec. 2006 , pp. 1706-1718.

[4] P. Gupta and P.R. Kumar, "The capacity of wireless networks," IEEE Trans. Inform. Theory, vol. 46, no. 2, Mar. 2000, pp. 388-404.

[5] K. Xu, X. Hong, and M. Gerla, "An ad hoc network with mobile backbones," in proc. IEEE ICC, Apr. 2002

[6] Xiaojiang Du, Moshen Guizani, Yang Xiao and Hsiao-Hwa Chen, "Two Tier Secure Routing Protocol for Heterogeneous Sensor Networks," IEEE transactions on Wireless Communications, Vol. 6, No. 9, September 2007, pp. 3395-3401.

[7] R. Jayaparvathy and R. Maheswar, "Maximization of network lifetime for sensor surveillance system," in Proc. NCSSC08, Anna University, Chennai, May 2008.

[8] J. Polastre, J. Hill and David Culler, "Versatile low power media access for wireless sensor networks," in proc. SenSys '04, pp. 95-107 (2004). 